E. Hesameddini, A. Fakharzadeh, M. Soleimanivareki, H. Alimorad /TJ MCS Vol .5 No.1 (2012) 67-74

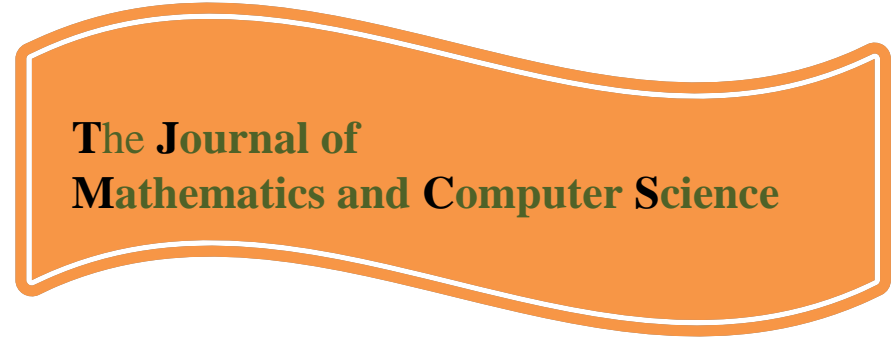

Available online at

\title{
http://www.TIMCS.com
}

The Journal of Mathematics and Computer Science Vol .5 No.1 (2012) 67-74

\section{Differential Transformation Method for Solving a Class of Nonlinear Optimal Control Problems}

\section{Esmail Hesameddini}

Department of Applied Mathematics, Faculty of Basic Sciences

Shiraz University of Technology, Shiraz, Iran

hesameddini@sutech.ac.ir

\section{Alireza Fakharzadeh Jahromi}

Department of Applied Mathematics, Faculty of Basic Science,

Shiraz University of Technology, Shiraz, Iran

a_fakharzadeh@sutech.ac.ir

\section{Mohammad Soleimanivareki ${ }^{1}$}

Department of Mathematics, Faculty of Basic Sciences, Islamic Azad university-science research Ayatollah Amoli branch,Amol, Iran.

m.soleimanivareki@sutech.ac.ir

Hajar Alimorad

Department of Applied Mathematics, Ph.D student

Shiraz University of Technology, Modarres Blvd

h.alimorad@sutech.ac.ir

Received: February 2012, Revised: November 2012

Online Publication: December 2012

\section{Abstract}

In this paper, the Differential Transformation Method (DTM) is suggested to solve a class of nonlinear optimal control problems. By applying the DTM, nonlinear two-point boundary value problem (TPBVP) which is derived from the Pontryagin's maximum principle, will be transformed to a sequence of linear time-invariant TPBVP's. Comparing the DTM to optimal homotopy perturbation method shows the efficiency and advantages of this method. In this method, a few iterations are required to find a suboptimal trajectory and control pair.

Keywords: Differential transformation method; Nonlinear optimal control problem; Pontryagin's maximum principle.

\footnotetext{
${ }^{1}$ Corresponding author.
} 
E. Hesameddini, A. Fakharzadeh, M. Soleimanivareki, H. Alimorad /TJ MCS Vol .5 No.1 (2012) 67-74

\section{Introduction.}

Application of optimal control theory and its modification have appeared in different fields such as economy, engineering, medicine, physics, aircraft systems, etc. (Bryson, 1986). Methods of solving nonlinear optimal control problems (OCPs) contains direct methods which converts the problem into a nonlinear programming one by using of the discretization or parameterization techniques, such as AVK and Tao methods, and indirect methods such as the use of Hamilton-Jacobi-Bellman (HJB) equation, dynamic programming, nonlinear two-point boundary value problem (TPBVP), or the Pontryagin's maximum principle ( Kirk, 2004; Jajrmi, 2010). The aim of this paper is to employ the DTM for solving a class of nonlinear OCPs. The results show that after only a few iterations it can be converged to the exact solution of the OCP, which proves that the suggested approach is very efficient in practice. The article is organized as follows. Section 2 describes the problem statement and definitions. The basic idea of DTM is explained in Section 3. In the following section, the DTM is employed to propose a new optimal control design strategy. Finally, in section 5, effectiveness of the proposed approach is verified by solving a numerical example.

\section{Basic definitions and theorems.}

Definition 2.1. If $u(x)$ is expressed as a Taylor series about a fixed point $x_{0}$, then it can be represented as:

$u(x)=\sum_{k=0}^{\infty} \frac{u^{k}\left(x_{0}\right)}{k !}\left(x-x_{0}\right)^{k}$.

If $u_{n}(x)$ is the $n$-partial sums of a Taylor series Eq. (1), then

$u_{n}(x)=\sum_{k=0}^{n} \frac{u^{k}\left(x_{0}\right)}{k !}\left(x-x_{0}\right)^{k}+R_{n}(x)$,

where $u_{n}(x)$ is called the $n-$ th Taylor polynomial of $u(x)$ about $x_{0}$ and $R_{n}(x)$ is the remainder term. If $U(k)$ is defined as:

$U(k)=\frac{1}{k !}\left[\frac{d^{k} u(x)}{d x^{k}}\right]_{x=x_{0}}$,

where $k=0,1,2 \ldots$ then (1) is reduced to:

$u(x)=\sum_{k=0}^{\infty} U(k)\left(x-x_{0}\right)^{k}$.

Moreover, the $n$ - partial sums of Taylor series (2) are also reduced to:

$u_{n}(x)=\sum_{k=0}^{n} U(k)\left(x-x_{0}\right)^{k}+R_{n}(x)$,

where $U(k)$ is called the differential transformation of function $u(x)$. If $x_{0}=0$, then it can be found that the concept of the one-dimensional differential transformation is derived from the Taylor series expansion.

Theorem 2.1. Suppose that $W(x), U(k)$ and $V(k)$ are differential transformation of the functions $w(x), u(x)$ and $v(x)$, respectively; then

a) If $w(x)=u(x) \pm v(x)$, then $W(k)=U(k) \pm V(k)$.

b) If $w(x)=\lambda u(x)$, then $W(k)=\lambda U(k)$ for each $\lambda \in \Re$.

c) Ifw $(x)=\frac{d^{m} u(x)}{d x^{m}}$, then $W(k)=\frac{(k+m) !}{k !} U(k+m)$.

d) If $w(x)=u(x) v(x)$, then $W(k) * v(k)=\sum_{l=0}^{k} U(l) V(k-l)$.

For more details and proofs, one can see (Abazari, 2010 ) and its references. 


\section{E. Hesameddini, A. Fakharzadeh, M. Soleimanivareki, H. Alimorad /TJ MCS Vol .5 No.1 (2012) 67-74}

Definition 2.2. Consider a function of two variables $w(x, y)$, and suppose that it can be represented as a product of two single-variable function, i.e., $w(x, y)=f(x) g(y)$. On the basis of the one-dimensional differential transform, this function can be represented as:

$$
w(x, y)=\sum_{i=0}^{\infty} F(i) x^{i} \sum_{j=0}^{\infty} G(j) y^{j}=\sum_{i=0}^{\infty} \sum_{j=0}^{\infty} W(i, j) x^{i} y^{j},
$$

whereW $(i, j)=F(i) G(j)$ is called spectrum of $w(x, y)$. Now, if $w(x, t)$ is an analytic and continuously differentiable function, then we have:

$W(k, h)=\frac{1}{k ! h !}\left[\frac{\partial^{k+h}}{\partial x^{k} \partial y} w(x, y)\right]\left(x_{0}, y_{0}\right)$,

where spectrum function $W(k, h)$ is the transformed function, which is also called T-function in brief. The differential inverse transform of $W(k, h)$ is defined as:

$w(x, y)=\sum_{k=0}^{\infty} \sum_{h=0}^{\infty} W(k, h)\left(x-x_{0}\right)^{k}\left(y-y_{0}\right)^{h}$.

By combining the relations (7) and (8), result in:

$w(x, y)=\sum_{k=0}^{\infty} \sum_{h=0}^{\infty} \frac{1}{k ! h !}\left[\frac{\partial^{k+h}}{\partial x} x^{k}{ }^{h} w(x, y)\right]_{\left(x_{0}, y_{0}\right)}\left(x-x_{0}\right)^{k}\left(y-y_{0}\right)^{h}$.

When $x_{0}$ and $y_{0}$ are taken in $(0,0)$, the relation (8) can be expressed as:

$w(x, y)=\sum_{k=0}^{\infty} \sum_{h=0}^{\infty} W(k, h) x^{k} y^{h}$.

From these definitions, it can be found that the concept of two-dimensional differential transform is derived by two-dimensional Taylor series expansion.

Theorem 2.2. Assume that $W(k, h), U(k, h)$ and $V(k, h)$ are differential transforms of the functions $w(x, y), u(x, y)$ and $v(x, y)$ respectively; then:

a) If $w(x, y)=u(x, y) \pm v(x, y)$, then $W(k, h)=U(k, h) \pm V(k, h)$.

b) If $w(x, y)=\frac{\partial^{r+s}}{\partial x^{r} \partial y^{s}} u(x, y)$, then $W(k, h)=\frac{(k+r) !(h+s) !}{k ! h !} U(k+r, h+s)$.

c) If $w(x, y)=u(x, y) v(x, y)$, then

$W(k, h)=\sum_{r=0}^{\infty} \sum_{s=0}^{\infty} U(r, h-s) V(k-r, s)$.

d) If $w(x, y)=\frac{\partial}{\partial x} u(x, t) \frac{\partial}{\partial y} v(x, y)$, then

$W(k, h)=\frac{\partial}{\partial x} u(x, t) * \frac{\partial}{\partial y} v(x, y)=\sum_{r=0}^{k} \sum_{s=0}^{h} \alpha \beta U(\alpha, s) V(r, \beta)$,

where, $\alpha=k-r+1, \beta=h-s+1$.

For more details and proofs see (Abazari, 2010) and its references.

3. Introducing the problem.

Consider a nonlinear control system described by:

$\dot{x}(t)=f(x(t))+B u(t) ; t \in\left[t_{0}, t_{f}\right], x\left(t_{0}\right)=x_{0}, x\left(t_{f}\right)=x_{f}$,

where $x \in \mathfrak{R}^{n}$ and $u \in \mathfrak{R}^{m}$ are the state and control vectors respectively. $t_{f}$ is finite, $f: R^{n} \rightarrow R^{n}$ 
is a nonlinear vector field, $\mathrm{B}$ is a constant matrix of appropriate dimension, and $x_{0}, x_{f} \in R^{n}$ are the initial and final state vectors, respectively. The aim is to find the optimal control law $u^{*}(t)$ which minimizes the following quadratic performance index subject to the described system (11):

$J=\frac{1}{2} \int_{t_{0}}^{t_{f}}\left(x^{T}(t) Q x(t)+u^{T}(t) R u(t)\right) d t$

where $Q \in R^{n \times n}$ and $R \in R^{m \times m}$ are positive semi-definite and positive definite matrices, respectively. According to the Pontryagin's maximum principle (Barron,1986), we have the following Hamiltonian function:

$H=f_{0}+\sum_{i=1}^{n} \lambda_{i} f_{i}=\frac{1}{2}\left\{\sum_{i=1}^{n} \sum_{j=1}^{n} q_{i j} x_{i} x_{j}+\sum_{k=1}^{m} \sum_{s=1}^{m} r_{k s} u_{k} u_{s}\right\}+\sum_{i=1}^{n} \lambda_{i}\left(\sum_{j=1}^{m} f_{i j} x_{j}+\sum_{k=1}^{m} b_{i k} u_{k}\right)$.

Moreover, the co-state equations are, $\dot{\lambda}_{i}(t)=-\frac{\partial H}{\partial x_{i}}=-\left\{\sum_{j=1}^{n} q_{i j} x_{j}+\sum_{j=1}^{n} \lambda j f_{i j}\right\}, i=0,1,2, \ldots, n$. If we reform these equations in matrix form, then, the co-state system of equations is presented as:

$\dot{\lambda}(t)=-Q x(t)-\left(\frac{\partial f(x(t))}{\partial x(t)}\right)^{T} \lambda(t)$.

Furthermore, by regarding the PMP for optimal control, the $u^{*}$ will be determined by solving the following equations:

$\frac{\partial H}{\partial u_{k}}=\left.\left(\sum_{s} r_{k s} u_{s}+\sum_{i} \lambda b_{i} b_{i k}\right)\right|_{u^{*}}=0$.

Thus, by substituting $u^{*}$ in matrix form of (15), i.e. $R u+B^{T} \lambda=0$, the optimal control low that minimizes $H$ is given by $u^{*}=-R^{-1} B^{T} \lambda$. In summary, the optimality conditions for problem (11) and (12) will be obtained as the following nonlinear TPVBP:

$\left\{\begin{array}{l}\dot{x}(t)=-B R^{-1} B^{T} \lambda(t)+F(x(t)), \\ \dot{\lambda}(t)=-Q x(t)-\left(\frac{\partial F(x(t))}{\partial x(t)}\right)^{T} \lambda(t), \\ x\left(t_{0}\right)=x_{0}, x\left(t_{f}\right)=x_{f} .\end{array}\right.$

\section{Optimal control design strategy via DTM.}

In this section, we apply the DTM for solving the nonlinear TPBVP (16). In this method system (16) will be transformed to:

$$
\begin{aligned}
& \left\{\begin{array}{l}
x_{1}(k+1)=\frac{1}{k+1}\left[\frac{-\lambda_{1}(k)}{\alpha_{1}^{2}}\right]+\left(\frac{-\left(\alpha_{3}-\alpha_{2}\right)}{\alpha_{1}}\right) x_{2}(k) x_{3}(k), \\
x_{2}(k+1)=\frac{1}{k+1}\left[\frac{-\lambda_{2}(k)}{\alpha_{2}^{2}}\right]+\left(\frac{-\left(\alpha_{1}-\alpha_{3}\right)}{\alpha_{2}}\right) x_{1}(k) x_{3}(k), \\
x_{3}(k+1)=\frac{1}{k+1}\left[\frac{-\lambda_{3}(k)}{\alpha_{3}^{2}}\right]+\left(\frac{-\left(\alpha_{2}-\alpha_{1}\right)}{\alpha_{3}}\right) x_{1}(k) x_{2}(k), \\
x(k+1)=\frac{1}{k+1}\left(-B R^{-1} B^{T} \lambda(k)+F(x(k))\right), \\
\lambda(k+1)=\frac{1}{k+1}\left(-Q x(k)-\left[\frac{\partial F(x(k))}{\partial x(k)}\right]^{T} \lambda(k)\right), \\
x\left(t_{0}\right)=x_{0}, x(t f)=x f ; u(k)=R^{-1} B^{T} \lambda(k) .
\end{array}\right.
\end{aligned}
$$


Also, for solving this system, we need $x(0), \lambda(0)$. But $x(0)$ is known since $x(0)=x\left(t_{0}\right)$ and for determining $u(0)$ we must firstly solve the following system:

$\left\{\begin{array}{l}\dot{x}(t)=-B R^{-1} B^{T} \lambda(t), \\ \dot{\lambda}(t)=-Q x(t) .\end{array}\right.$

The algorithm that will be used to solve this system is as follows:

Algorithm:

Step1. Obtain $x\left(t_{0}\right), \lambda\left(t_{0}\right)$.

Step2. Calculate $x(k+1), \lambda(k+1) ; \quad k=1,2, \ldots$ from solving (17).

Step3. Solve this problem until it gets accurate and iteration enough.

Step4. Determine $u(k)$ from $u(k)=R^{-1} B^{T} \lambda(k)$.

Step5. Calculate $x^{*}(t)=\sum_{k=0}^{N} \frac{x(k)}{k !} t^{k}, u^{*}(t)=\sum_{k=0}^{N} \frac{u(k)}{k !} t^{k}$.

5. Numerical examples.

In this section, we solve the Euler's equations for the angular velocities associated with the optimal maneuvers of a rigid symmetric spacecraft with DTM. This problem has been solved by optimal homotopy perturbation method in (Jajarmi, 2011). This equation is given by:

$$
\dot{x}(t)=\left[\begin{array}{l}
\dot{x}_{1}(t) \\
\dot{x}_{2}(t) \\
\dot{x}_{3}(t)
\end{array}\right]=\left[\begin{array}{c}
-\frac{\left(\alpha_{3}-\alpha_{2}\right)}{\alpha_{1}} x_{2}(t) x_{3}(t) \\
-\frac{\left(\alpha_{1}-\alpha_{3}\right)}{\alpha_{2}} x_{1}(t) x_{3}(t) \\
-\frac{\left(\alpha_{2}-\alpha_{1}\right)}{\alpha_{3}} x_{1}(t) x_{2}(t)
\end{array}\right]+\left[\begin{array}{ccc}
\frac{1}{\alpha_{1}} & 0 & 0 \\
0 & \frac{1}{\alpha_{2}} & 0 \\
0 & 0 & \frac{1}{\alpha_{3}}
\end{array}\right]\left[\begin{array}{l}
u_{1}(t) \\
u_{2}(t) \\
u_{3}(t)
\end{array}\right],
$$

Where $x_{1}, x_{2}$ and $x_{3}$ are angular velocities of spacecraft, $u_{1}, u_{2}$ and $u_{3}$ are control torques, $\alpha_{1}=86.24 \mathrm{kgm}^{2}, \alpha_{2}=85.07 \mathrm{kgm}^{2}$ and $\alpha_{3}=113.59 \mathrm{kgm}^{2}$ are spacecraft principle inertia. Also, $t_{0}=0$, $t_{f}=100, Q=0, R=I_{3}, x_{1}(0)=0.01, x_{2}(0)=0.005, x_{3}(0)=0.001, x_{1}(100)=x_{1}(100)=x_{1}(100)=0$.

According to the Pontryagin's maximum principle, the following nonlinear TPBVP system is obtained:

$$
\begin{aligned}
& \dot{x}(t)=\left[\begin{array}{c}
\dot{x}_{1}(t) \\
\dot{x}_{2}(t) \\
\dot{x}_{3}(t)
\end{array}\right]=\left[\begin{array}{c}
-\frac{\left(\alpha_{3}-\alpha_{2}\right)}{\alpha_{1}} x_{2}(t) x_{3}(t) \\
-\frac{\left(\alpha_{1}-\alpha_{3}\right)}{\alpha_{2}} x_{1}(t) x_{3}(t) \\
-\frac{\left(\alpha_{2}-\alpha_{1}\right)}{\alpha_{3}} x_{1}(t) x_{2}(t)
\end{array}\right]-\left[\begin{array}{c}
\frac{\lambda_{1}(t)}{\alpha_{1}^{2}} \\
\frac{\lambda_{2}(t)}{\alpha_{2}^{2}} \\
\frac{\lambda_{3}(t)}{\alpha_{3}^{2}}
\end{array}\right], \\
& \dot{\lambda}(t)=\left[\begin{array}{c}
\dot{\lambda}_{1}(t) \\
\dot{\lambda}_{2}(t) \\
\dot{\lambda}_{3}(t)
\end{array}\right]=\left[\begin{array}{l}
\frac{\left(\alpha_{1}-\alpha_{3}\right)}{\alpha_{2}} x_{3}(t) \lambda_{2}(t)+\frac{\left(\alpha_{2}-\alpha_{1}\right)}{\alpha_{3}} x_{2}(t) \lambda_{3}(t) \\
\frac{\left(\alpha_{3}-\alpha_{2}\right)}{\alpha_{1}} x_{3}(t) \lambda_{1}(t)+\frac{\left(\alpha_{2}-\alpha_{1}\right)}{\alpha_{3}} x_{1}(t) \lambda_{3}(t) \\
\frac{\left(\alpha_{3}-\alpha_{2}\right)}{\alpha_{1}} x_{2}(t) \lambda_{1}(t)+\frac{\left(\alpha_{1}-\alpha_{3}\right)}{\alpha_{2}} x_{1}(t) \lambda_{2}(t)
\end{array}\right] ; x(0)=\left[\begin{array}{l}
0.010 \\
0.005 \\
0.001
\end{array}\right], \lambda(0)=\left[\begin{array}{l}
0.7437337601 \\
0.3618452452 \\
0.1290268810
\end{array}\right.
\end{aligned}
$$

By solving this system with DTM one obtain: 
E. Hesameddini, A. Fakharzadeh, M. Soleimanivareki, H. Alimorad /TJ MCS Vol .5 No.1 (2012) 67-74

$$
\left\{\begin{array}{l}
\lambda_{1}(k+1)=\frac{1}{k+1}\left\{-\frac{\left(\alpha_{1}-\alpha_{3}\right)}{\alpha_{2}} \sum_{l=0}^{k} x_{3}(k-l) \lambda_{2}(l)-\frac{\left(\alpha_{2}-\alpha_{1}\right)}{\alpha_{3}} \sum_{l=0}^{k} x_{2}(k-l) \lambda_{3}(l)\right\} \\
\lambda_{2}(k+1)=\frac{1}{k+1}\left\{-\frac{\left(\alpha_{3}-\alpha_{2}\right)}{\alpha_{1}} \sum_{l=0}^{k} x_{3}(k-l) \lambda_{1}(l)-\frac{\left(\alpha_{2}-\alpha_{1}\right)}{\alpha_{3}} \sum_{l=0}^{k} x_{1}(k-l) \lambda_{3}(l)\right\} \\
\lambda_{3}(k+1)=\frac{1}{k+1}\left\{-\frac{\left(\alpha_{3}-\alpha_{2}\right)}{\alpha_{1}} \sum_{l=0}^{k} x_{2}(k-l) \lambda_{1}(l)-\frac{\left(\alpha_{1}-\alpha_{3}\right)}{\alpha_{2}} \sum_{l=0}^{k} x_{1}(k-l) \lambda_{2}(l)\right\} \\
x_{1}(k+1)=-\frac{\left(\alpha_{3}-\alpha_{2}\right)}{\alpha_{1}} \sum_{l=0}^{k} x_{2}(k-l) x_{3}(l)-\frac{1}{\alpha_{1}^{2}} \lambda_{1}(k), \\
x_{2}(k+1)=-\frac{\left(\alpha_{1}-\alpha_{3}\right)}{\alpha_{2}} \sum_{l=0}^{k} x_{1}(k-l) x_{3}(l)-\frac{1}{\alpha_{2}^{2}} \lambda_{2}(k), \\
x_{3}(k+1)=-\frac{\left(\alpha_{2}-\alpha_{1}\right)}{\alpha_{3}} \sum_{l=0}^{k} x_{1}(k-l) x_{2}(l)-\frac{1}{\alpha_{3}^{2}} \lambda_{3}(k) .
\end{array}\right.
$$

And

$$
u(k)=-\left[\begin{array}{c}
\frac{\lambda_{1}(k)}{\alpha_{1}} \\
\frac{\lambda_{2}(k)}{\alpha_{2}} \\
\frac{\lambda_{3}(k)}{\alpha_{3}}
\end{array}\right] ; t \in[0,100] .
$$

Simulation curves of the state trajectories and control laws, computed by the suggested DTM, have been shown in figure1. It shows that the obtained solutions by DTM are closely with those of collocation methods and we estimate these without any high computations.
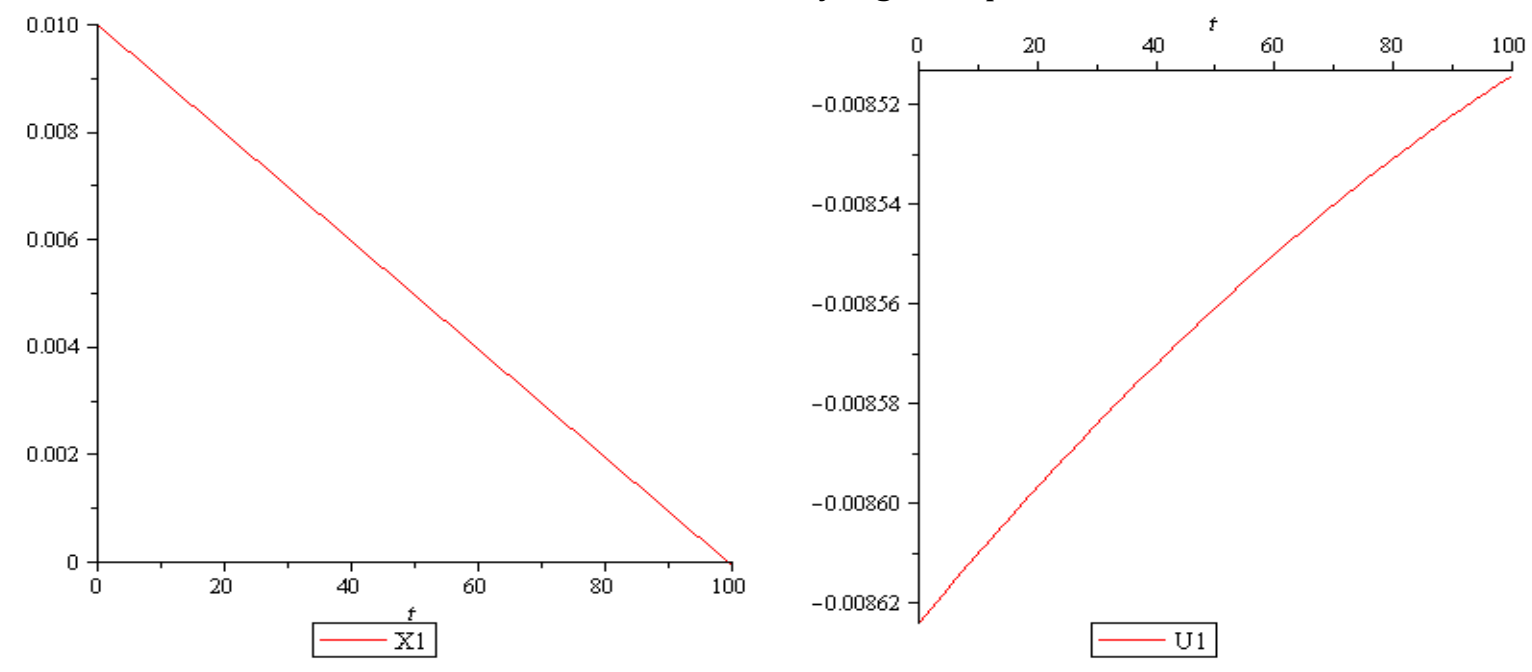
E. Hesameddini, A. Fakharzadeh, M. Soleimanivareki, H. Alimorad /TJ MCS Vol .5 No.1 (2012) 67-74
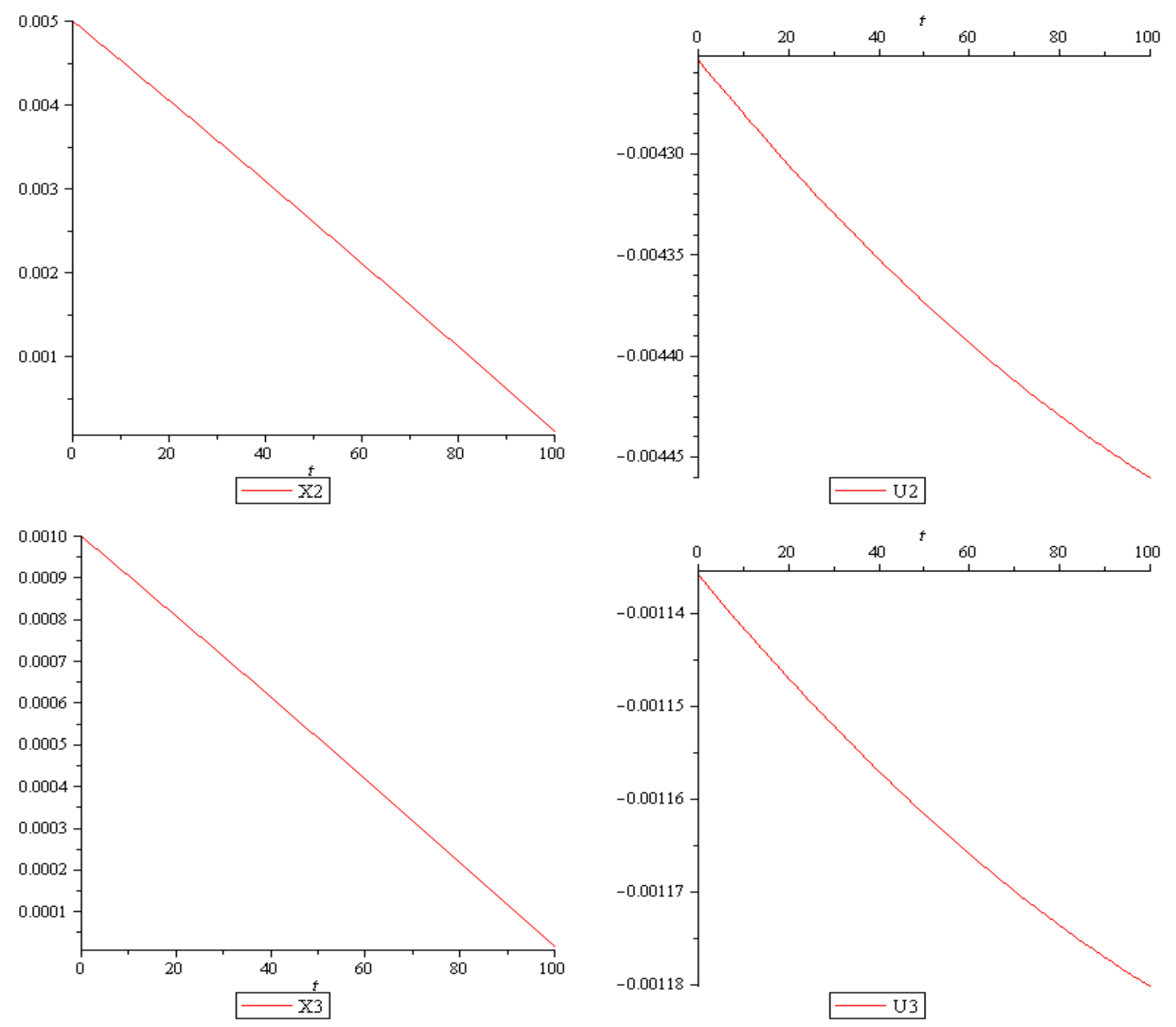

Figure1: Simulation curves of $x_{1}(t), x_{2}(t), x_{3}(t), u_{1}(t), u_{2}(t), u_{3}(t)$ computed by DTM

\section{Conclusions.}

In this paper, we introduced ADM for solving the nonlinear optimal control problems. This method has some advantages over the OHPM that was presented in (Jajarmi,2011). Firstly it doesn't need high computations, secondly in OHPM auxiliary function depends on several unknown constants, but this method has a fixed process. Also, the required time to gain the acceptable solution in this method is less than that of others.

Acknowledgment. The third authors would like to sincerely thank the Islamic Azad universityscience research Ayatollah Amoli branch for their particularly financial support.

\section{References.}

[1] A. E. Bryson, Applied linear Optimal Control: Examples and Algorithm, Cambridge University Press, UK (2002).

[2] A. Jajrmi, H. Ramezanpour, A. Sargolzaei, P. Shafaei, Optimal control of Nonlinear systems Using the Homotopy Perturbation Method: Infinite Horizon Case, International Journal of Digital Technology and its Applications, 4 (2010), N .9,114-122.

[3] A. Jajarmi, N. Pariz, A. Vahidian Kamyad, S. Effati, A Highly Computational Efficient Method to Solve Nonlinear optimal Control Problems, Scientia Iranica, Transactions D: computer \& Engineering and electrical Engineering 2011,759-766. 


\section{E. Hesameddini, A. Fakharzadeh, M. Soleimanivareki, H. Alimorad /TJ MCS Vol .5 No.1 (2012) 67-74}

[4] N. Barron and R. Jensen, The Pontryagin maximum Principle form Dynamic programming and Viscosity Solutions to First-partial Differntial Equations, Transections AMS, 298 (1986), 635-641.

[5] D. E. Kirk, Optimal Control Theory An Introduction, Dover Publications, Inc. Mineola, New York (2004).

[6] R. Abazari, Numerical Study of Some Coupled PDEs by Using differential Transformation Method, Word Academy of Science, Engineering and Technology ,66 (2010),52-59. 